\title{
Nuclear Mobility and Mitotic Chromosome Binding Similarities between Pioneer Transcription Factor FoxA and Linker Histone $\mathrm{Hl}$
}

\author{
K.S. Zaret, ${ }^{1}$ J.M. CARAvacA, ${ }^{1}$ A. Tulin, ${ }^{2}$ And T. SEKIYA ${ }^{1}$ \\ ${ }^{1}$ Epigenetics Program and Institute for Regenerative Medicine, Department of Cell and Developmental \\ Biology, University of Pennsylvania School of Medicine, Philadelphia, Pennsylvania 19107; \\ ${ }^{2}$ Fox Chase Cancer Center, Philadelphia, Pennsylvania 19111 \\ Correspondence: zaret@upenn.edu
}

\begin{abstract}
There exists a hierarchy by which transcription factors can engage their target sites in chromatin, in that a subset of factors can bind transcriptionally silent, nucleosomal DNA, whereas most factors cannot, and this hierarchy is reflected, at least in part, in the developmental function of the factors. For example, transcription factors possessing the Forkhead box (Fox) DNAbinding domain contain an overall fold resembling that of linker histone and thus are structured to bind DNA, site specifically, in a nucleosomal context. Where tested, Fox factors bind early in the developmental or physiological activation of target genes, thereby enabling the binding of other factors that cannot engage chromatin on their own. To investigate the basis for early chromatin binding, we have used fluorescence recovery after photobleaching (FRAP) to analyze the mobility, in the live cell nucleus, of FoxA factors in comparison to linker histone and other transcription factors. We have further analyzed the factors for their ability to bind to chromatin in mitosis and thereby serve as epigenetic marks. The results indicate that the "pioneer" features of FoxA factors involve various chromatin-binding parameters seen in linker histones and that distinguish the factors with respect to their regulatory and mechanistic functions.
\end{abstract}

The structures of the DNA-binding domain (DBD) of transcription factors can provide important clues about their mechanistic and biological functions. For example, certain DBDs contain protein segments that pass around the DNA helix, preventing the binding of proteins to the other side of the DNA. Binding of these factors to chromatin would be predicted to occur at sites free of nucleosomes. In contrast, other factors can bind stably to one face of the DNA helix, which could allow proteins to be bound to the other side. FoxA factors fall in the latter class because their "winged-helix" DBD binds as a monomer simultaneously to the major and minor groove sites on one side of the DNA (Clark et al. 1993), thus allowing binding of the factor to its target sites on nucleosomes (Cirillo et al. 1998; Cirillo and Zaret 1999). As expected, rotation of the Fox target DNA sequence into the nucleosome impairs specific binding (Sekiya et al. 2009). The three FoxA genes in mammals are among the 43 genes that encode Fox proteins (Hannenhalli and Kaestner 2009), among which FoxE and FoxO have also been shown to bind target-site nucleosomes (Cuesta et al. 2007; Hatta and Cirillo 2007). The overall fold of the FoxA DBD (Clark et al. 1993) and that of most Fox proteins are remarkably similar to that of linker histone (Ramakrishnan et al. 1993), which binds nonspecifically to DNA and avidly to nucleosomes (Zhou et al. 1998). Notably, the structure of the Fox DBD and its ability to bind nucleosomal DNA is consistent with the mechanistic and biological functions of the factor class. That is, Fox factors often function in early developmental contexts and in differentiated cells by opening up local chromatin domains and enabling or enhancing the ability of other factors to bind (Gualdi et al. 1996; Cirillo et al. 2002; Lee et al. 2005). In this chapter, we investigate the mechanistic basis by which Fox factors, in comparison to other factors and linker histone, bind and move in living nuclear chromatin and in mitotic cells. On the basis of these physical characteristics, we find that in addition to nucleosome binding, FoxA factors fall in an intermediate class between linker histones and other transcription factors and that this classification helps explain the factors' biological activity.

\section{CORRELATION BETWEEN HIGH NUCLEOSOME-BINDING AFFINITY AND LOW NUCLEAR MOBILITY}

In a previous study, we investigated how the nucleosome-binding ability of diverse transcription factors, each with different DNA-binding domain structures, relates to the ability of the factors to move in the nucleus (Sekiya et al. 2009). Nuclear mobility is measured by FRAP, wherein a laser is first used to bleach a spot in the nucleus containing green fluorescent protein (GFP)-tagged protein. The movement of unbleached protein into the bleached area is then quantitated by time-lapse confocal microscopy (McNally et al. 2000; Misteli et al. 2000). FRAP of core histones has been determined to be relatively slow, taking many minutes (Kimura and Cook 2001), whereas the movement of most transcription factors is fast, usually within seconds (Phair et al. 2004). FRAP of a transcription factor could relate to its relative abundance in the nucleus, its off-rate from occupied sites in chromatin, its mobility 
(i.e., diffusion) in the nucleoplasm, and other parameters (Hager et al. 2009). Together, these parameters define how a transcription factor scans nuclear DNA. Given that general, nonspecific chromatin binding must be an important parameter governing nuclear DNA scanning, we compared nuclear mobility to the ability of various transcription factors to bind nonspecifically to nucleosomal DNA. For these studies, we used two different model nucleosomes reconstituted in vitro with purified, recombinant core histones. The models contained either the NCP147 $\alpha$-satellite sequence used for nucleosome crystallization (Luger et al. 1997) or the 5S rDNA sequence (Simpson et al. 1985); both sequences position the histone octamer on the DNA, thus yielding relatively uniform nucleosomal preparations that can be used in quantitative studies. The nucleosomes were used in electromobility shift titration assays with purified, recombinant FoxA1 protein (wingedhelix DBD), c-Myc (basic helix-loop-helix DBD), GATA-4 (Zn-finger DBD), HMGB1 (high mobility group DBD), and NF-1 (4x cys $+\alpha$-helix DBD). Because both nucleosome substrates lacked target sites for any of the factors, we determined the factors' relative affinity for nonspecific binding to the nucleosomal DNA. These data were compared with the relative affinities for binding to the same free DNAs, without core histones, and with the nuclear mobility of each of the factors, as determined by transfecting cells with the same proteins in GFP-tagged forms, normalizing for expression levels, and performing FRAP experiments.

Using these approaches, we discovered a clear inverse correlation - that is, the greater the nucleosome-binding affinity of the factor, the slower its nuclear mobility. Conversely, the poorer the nucleosome-binding affinity, the faster its nuclear mobility (Sekiya et al. 2009). This correlation was stronger than associating nuclear mobility with relative affinities for free DNA. Notably, of all the factors tested, FoxA1 bound nucleosomal DNA more avidly and exhibited much slower nuclear mobility (Sekiya et al. 2009), and slower mobility than most other factors tested in a separate study (Phair et al. 2004). We then tested point mutants of FoxA1 in the DBD helixturn-helix motif that binds specific bases in the DNA major groove and thus should impede specific binding to DNA. We also tested point mutants of FoxA1 in a DBD "wing" domain that binds nonspecifically to the phosphodiester backbone along the minor groove and thus should impede nonspecific binding to DNA. The mutations had the expected effects on DNA and nucleosome binding; that is, the specific site-binding mutant lost discrimination for the FoxA1 target sequence but retained general DNAand nucleosome-binding characteristics, whereas the nonspecific binding mutant exhibited a greatly diminished DNA- and nucleosome-binding capacity but still could discriminate between a specific and a nonspecific target. In FRAP assays, the specific site-binding mutant exhibited a minor increase in nuclear mobility, whereas the nonspecific binding mutant exhibited a major increase in nuclear mobility (Sekiya et al. 2009). Taking all of the results together, we conclude that the nonspecific nucleosomebinding capacity of a protein provides the major basis for its nuclear mobility. Previous studies had drawn the conclusion that "nonspecific DNA sites" are the primary determinant of chromatin binding detected by FRAP (Karpova et al. 2004; Hinow et al. 2006; Mueller et al. 2008), and we suggest that such nonspecific sites are nucleosomal in nature, not in free DNA.

Finally, we used a "half nuclear FRAP assay" (Phair et al. 2004) to directly observe transcription factors traversing the nucleus. Although fluorescence recovery of GFPc-Myc occurred nearly uniformly in the bleached half-nucleus, recovery of GFP-FoxA1 occurred first near the edge of the unbleached half and then slowly spread across the bleached half (Sekiya et al. 2009). These data suggest that fast-moving factors such as c-Myc exchange readily with the nucleoplasm and can diffuse within it, whereas slow-moving factors such as FoxA1 that bind nucleosomal DNA more avidly, exchange more poorly with the nucleoplasm and move laterally along the chromatin. We speculated that strong nucleosome binding and slow nuclear movement allow FoxA to locate high-affinity target sites in highly compacted chromatin, perhaps explaining the aforementioned pioneer functions of FoxA proteins in early developmental gene activation and in enabling other factors to enter silent chromatin sites.

\section{FOXA CARBOXY-TERMINAL DOMAIN DISTINGUISHES TRANSCRIPTION FACTOR MOBILITY FROM THAT OF LINKER HISTONE}

The above studies left unanswered how the chromatin mobility of FoxA factors could be modulated and its relationship to the mobility of linker histones, with which the FoxA DBD shares structural features. To this end, we first tested amino- and carboxy-terminal deletion mutants of FoxA proteins in the FRAP assay, starting with GFPFoxA1 and GFP-FoxA2. The amino-terminal regions of FoxA1 and FoxA2 possess a trans-activation domain that can be independently attached to a different DNA-binding domain and elicit trans-activation (Pani et al. 1992); however, putative coregulators have not been defined. As seen in Figure 1A,B, FoxA1 or FoxA2 deleted for their aminoterminal regions exhibited a fluorescence recovery that is identical to that of wild-type FoxA1 or FoxA2, respectively $\left(t_{1 / 2} \sim 14 \mathrm{sec}\right.$; cf. red and blue circless). Thus, any putative coregulators interacting with the amino-terminal domain do not affect FoxA nuclear mobility. These assays were conducted on transfected H2.35 hepatic cells (Zaret et al. 1988) as described (Sekiya et al. 2009), with comparable fluorescence levels of the expressed, GFP-tagged proteins (data not shown).

In earlier studies, we had found that the carboxy-terminal domain of FoxA1 independently interacts with core histone octamers and is necessary, in vitro, for FoxA1 to create hypersensitivity of an underlying nucleosome in compacted chromatin (Cirillo et al. 2002). We also found that FoxA1 does not interact with the core histone tails and thus would interact with the exposed globular domains of the histone octamer. It is known that the exposed globular domains of the histones stabilize the higher-order 
A

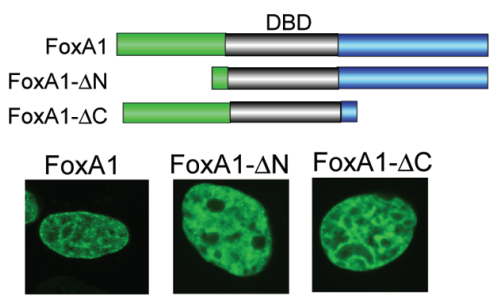

B

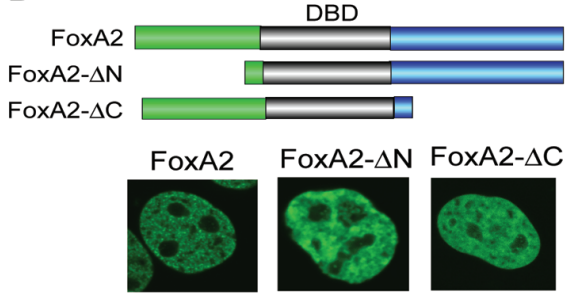

C

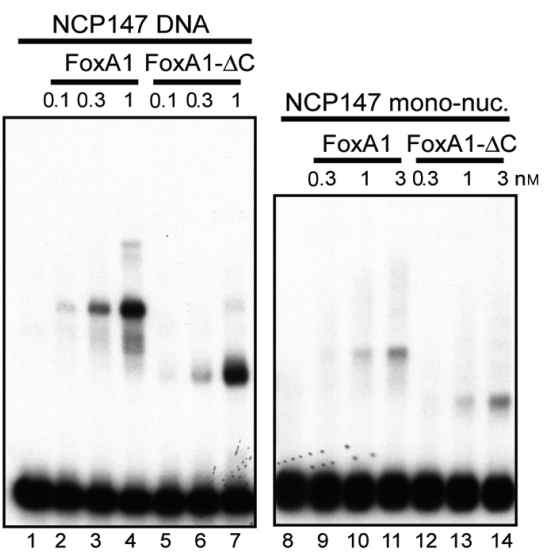

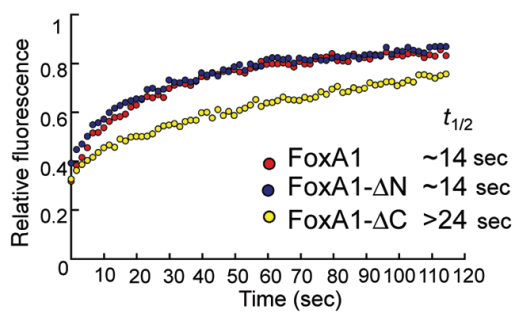
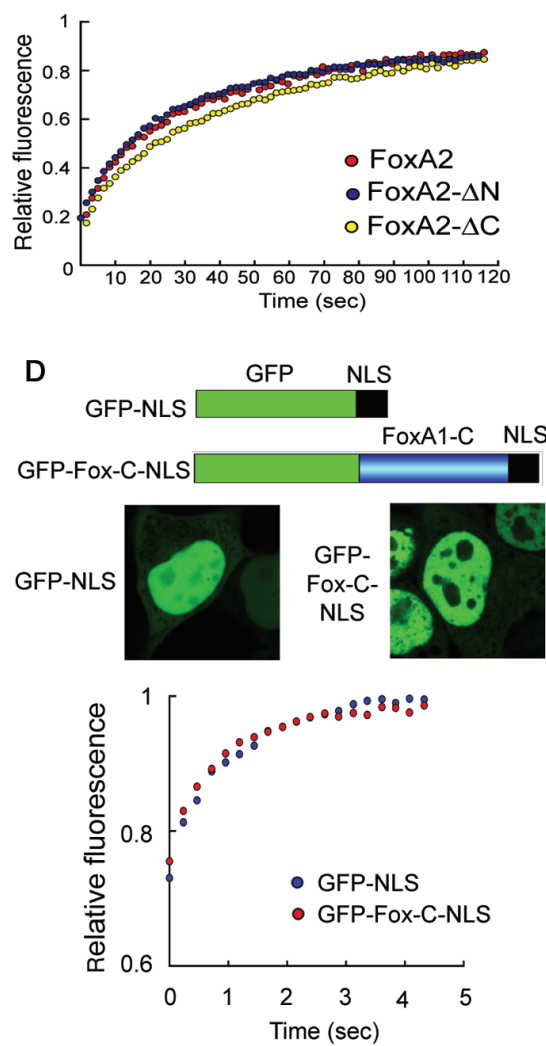

Figure 1. FoxA carboxy-terminal domain enhances FoxA1 and FoxA 2 mobility in the nucleus. ( $A, B$, top left $)$ Schematic representations of amino- and carboxy-terminal deletion mutants of FoxA1 and FoxA2. (A,B, Bottom left) Expected nuclear localization of GFP-fusion proteins transfected into $\mathrm{H} 2.35$ hepatic cells at $37^{\circ} \mathrm{C}$. $(A, B$, Right $)$ FRAP of GFP-fusion proteins, as indicated. In each nucleus, a $0.5-$ $\mu \mathrm{m}$-diameter circle area was bleached, and the kinetics of the average fluorescence intensity in the area was quantified and plotted. Values represent means from at least 10 cells. $t_{1 / 2}$ values are shown in $A$. (C) Electrophoretic mobility shift assay (EMSA) of NCP147 DNA and mononucleosomes, with $1 \mathrm{~nm}$ purified FoxA1 and FoxA1- $\Delta \mathrm{C}$ (for details of protein production and the assay, see Sekiya et al. 2009). ( $D$, top panel) Schematic representations of GFP fused to the SV40 nuclear localization signal (NLS) and the same but with the FoxA1 carboxy-terminal domain inserted between GFP and the NLS. (D, Lower panel) FRAP assays showing that the addition of the carboxy-terminal domain to GFP-NLS does not alter its fluorescence recovery kinetics.

structure of chromatin by promoting internucleosome interactions along the chromatin fiber (Schalch et al. 2005). We thus postulated that the carboxy-terminal domain, targeted to a nucleosome by the FoxA DBD, interacts with the core histone globular domains and disrupts local internucleosomal interactions, thereby creating a hypersensitive site (Cirillo et al. 2002).

In this context, it was interesting to discover that deletions of the carboxy-terminal domain had a profound effect on FoxA factors' nuclear mobility. Specifically, carboxy-terminal deletion of FoxA1 (FoxA1- $\Delta$ C) resulted in a markedly slower FRAP (Fig. 1A, $t_{1 / 2}>24 \mathrm{sec}$; yellow circles). CTD-deleted FoxA2 also had reduced FRAP
(Fig. 1B, yellow circles), albeit less than that caused by the FoxA1-CTD deletion. These results indicate that the carboxy-terminal domain enhances an intrinsically very slow mobility of the FoxA DBD in the nucleus.

We tested by EMSA whether the slower FRAP of FoxA $1-\Delta \mathrm{C}$ could be due to an increase in nonspecific DNA or nucleosome binding; however, FoxA1- $\Delta \mathrm{C}$ bound NCP147 free DNA and mononucleosomes similar to FoxA1 (Fig. 1C). We note that the DBD of FoxA2, deleted for its amino- and carboxy-terminal domains, functions as a dominant negative mutant, efficiently suppressing a variety of endogenous FoxA target genes (Vallet et al. 1995). Thus, the CTD is not needed for target sequence speci- 
ficity in vivo. Fusion of the isolated CTD to GFP, along with a nuclear localization signal (NLS), showed the same FRAP as GFP fused to the NLS alone (Fig. 1D), which diffuses randomly (Phair et al. 2004). Thus, the FoxA1CTD is insufficient to affect GFP mobility on its own.

Interestingly, the fluorescence recovery of FoxA1- $\Delta \mathrm{C}$ approached that of linker histone H1o fused to GFP (Fig. 2A) (note that H1o recovery was as previously reported by Misteli et al. 2000). Considering the structural similarity between the FoxA-DBD and the linker histone globular domain (Clark et al. 1993; Ramakrishnan et al. 1993), we speculated that a function of the CTD is to increase the
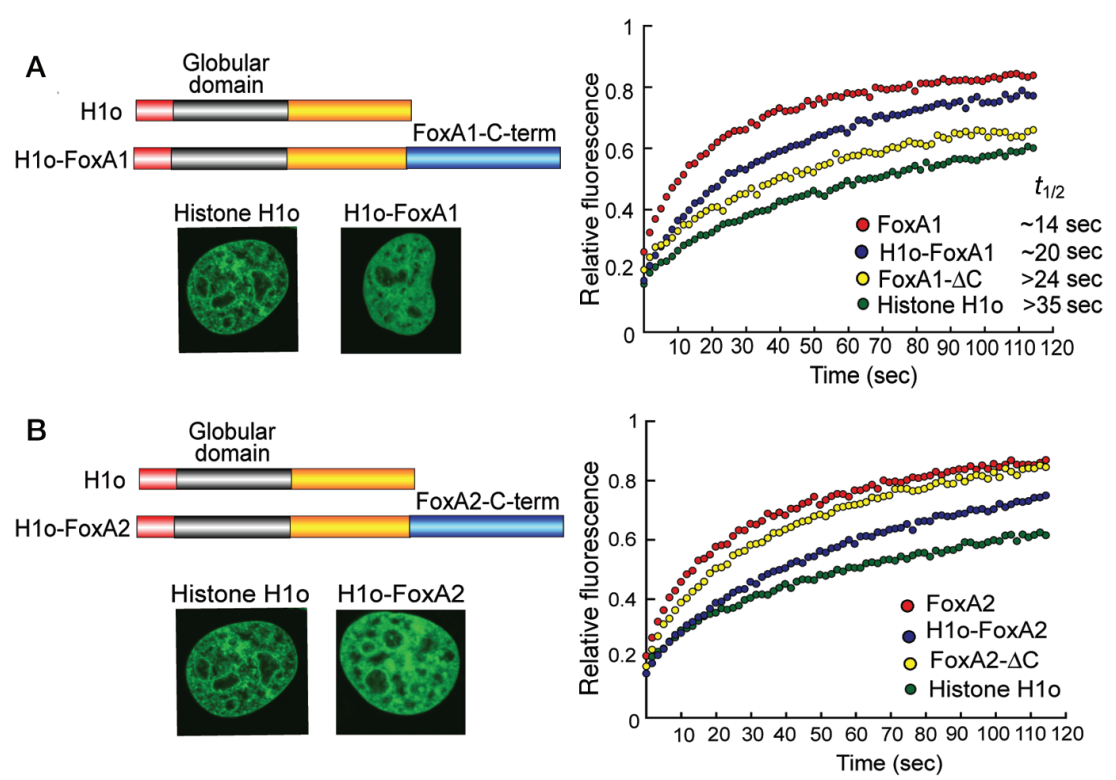

C
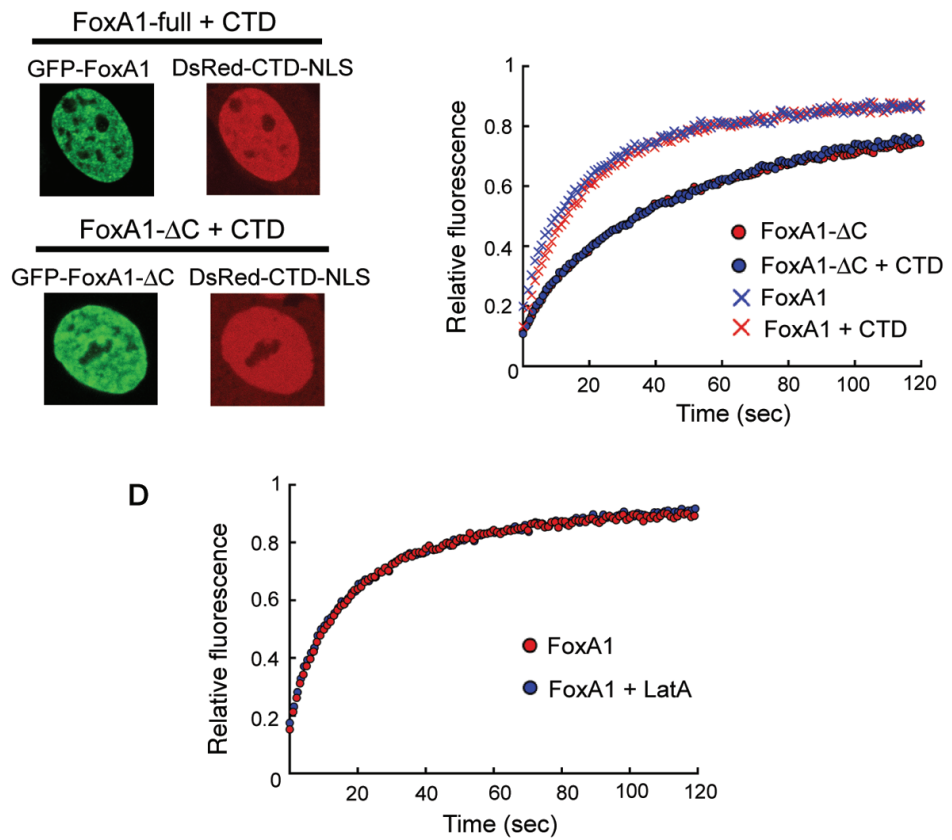

Figure 2. Carboxy-terminal domain of FoxA1 and FoxA2 helps to distinguish the transcription factors from linker histone, with regard to nuclear mobility. ( $A, B$, top left) Schematic representations of histone H1o (green circles in FRAP display at right) and H1o-FoxA1 carboxy-terminal domain chimeras (blue circles in FRAP display at right). (A,B, bottom left) Nuclear localization of GFP-fusion proteins. (A,B, Right) Display of FRAP data as indicated, including analysis of FoxA1 and FoxA2 (red circles in FRAP display) and FoxA1- $\Delta \mathrm{C}$ and FoxA2- $\Delta \mathrm{C}$ (yellow circles in FRAP display) in parallel. Deletion of the FoxA1 carboxy-terminal domain decreases nuclear mobility of the transcription factor to levels close to that of GFP-H1o. Deletion of the FoxA2 carboxy-terminal domain has a more modest effect. Conversely, addition of FoxA1's carboxy-terminal domain to H1o increases the latter's nuclear mobility, closer to that seen by the transcription factor; again, the FoxA2 carboxy-terminal domain has a more modest effect. $(C)$ Fluorescence recovery kinetics of GFP-FoxA1 proteins as indicated, in the presence or absence of DsRed-monomer fused FoxA1-CTD-NLS. Carboxyterminal domain does not complement the defect of the FoxA1- $\Delta \mathrm{C}$ in trans. $(D)$ Fluorescence recovery kinetics of GFP-FoxA1 is not affected by cell treatment with latrunculin A (LatA), which elicits, indicating that gross changes in nuclear architecture elicited by the drug (data not shown; see Nunez et al. 2008) do not affect how FoxA1 interacts with chromatin in vivo. 
mobility of FoxA1 in chromatin so that it does not move like linker histone. To test this, we attached the FoxA1 CTD to the carboxyl end of GFP-H1o (Fig. 2A). Strikingly, the recovery of GFP-H1o became significantly faster, closer to that of FoxA1 (Fig. 2A). Attachment of the FoxA2 CTD to GFP-H1o also enhanced FRAP (Fig. 2B), albeit less so than by the FoxA1 CTD.

Although the observed effect of the FoxA1 CTD on histone H1o might be the result of an artificial polypeptide attachment to the H1o carboxyl terminus, we note that the carboxyl attachment of GFP, which is $\sim 1.5$ times larger than the FoxA1 CTD, was argued by another study (Misteli et al. 2000) not to affect the mobility of histone H1o. More significantly, the small difference in effects of the FoxA1-CTD and FoxA2-CTD attachments to H1o (Fig. $2 \mathrm{~A}, \mathrm{~B})$ corresponds well to the small difference in effects of their deletion from the respective FoxA proteins (Fig. 1A,B). These results indicate that the CTD of FoxA1 helps distinguish the intrinsically slow nuclear mobility of the transcription factor's DBD from that of the structurally similar linker histone.

Coexpression of GFP-FoxA1 with the isolated FoxA1 carboxy-terminal domain, the latter linked to an NLS and DsRed to confirm nuclear localization, did not increase the mobility of FoxA1- $\Delta \mathrm{C}$ in trans (Fig. 2C, circles), together indicating that the CTD must affect FoxA1 in cis. Coexpression of DsRed-CTD-NLS also did not affect the mobility of wild-type FoxA1 (Fig. 2C, crosses); a positive result would have suggested competition for CTD binding to a limiting mobilization factor. To assess whether nuclear actin architecture could affect FoxA1 mobility, as seen for LSD1 (Nunez et al. 2008), we treated cells for $30 \mathrm{~min}$ with LatA at $1 \mu \mathrm{M}$. Although we observed irregular-shaped nuclei (data not shown), indicating the effectiveness of the LatA treatment, the FRAP of the FoxA1-full was not affected (Fig. 2D). Taken together, the results show that FoxA1 and FoxA2 contain a distinct, $c i s$-acting amino acid domain that confers nuclear mobility on an intrinsically slow-moving DNAbinding domain, the winged-helix motif.

\section{INVERSE RELATIONSHIP BETWEEN SALT ELUTION PROFILES FROM NUCLEI AND THE NUCLEAR MOBILITY OF FOXA1 AND H10}

A concern with FRAP studies is that differences in observed mobility could reflect differences in factors' reactions to potential obstacles to nuclear diffusion that might be created by ultraviolet (UV) laser photobleaching. To address this, we compared various GFP-fusions and native proteins for their ability to be salt-eluted from nonilluminated nuclei. Endogenous FoxA1 eluted mostly at $250 \mathrm{~mm}$, with some spread to higher and lower salt concentrations (Fig. 3, lane 2), whereas H1o and GFP-H1o eluted at 500 mM (Fig. 3, lanes 5 and 6). GFP-FoxA1 and GFP-FoxA1$\Delta \mathrm{N}$ also eluted mainly at $250 \mathrm{~mm}$, with a bit more spread than endogenous FoxA1 (Fig. 3, lanes 1 and 3). Importantly, however, FoxA1- $\Delta \mathrm{C}$ shifted its main elution peak to $500 \mathrm{~mm}$ (Fig. 3, lane 4), like linker histone. The increase in nuclear binding inversely parallels the slower FRAP following CTD deletion. Furthermore, the elution pattern of

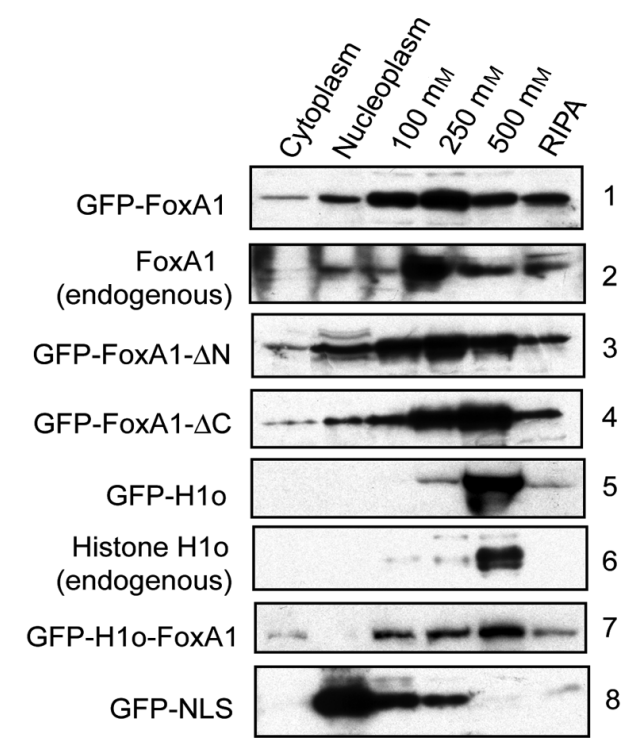

Figure 3. Salt elution profiles from nuclei inversely relate to the nuclear mobility of FoxA1 and H1o. H2.35 hepatic cells, not transfected or transfected with plasmids that express GFP-fusion proteins, were fractionated into cytoplasmic, nucleoplasmic, and NP-40-resistant extracts. The latter were eluted with 100, 250, and $500 \mathrm{~mm} \mathrm{NaCl}$ and RIPA buffer as indicated. Fifteen micrograms of protein from each fraction was analyzed by sodium dodecyl sulfate-polyacrylamide gel electrophoresis (SDS-PAGE) and western blotting.

the H1o-FoxA1-CTD chimera shifted partly to a lower salt than that of $\mathrm{H} 1 \mathrm{o}$ and also eluted with a different low-salt buffer (Fig. 3, lane 7; RIPA [radioimmunoprecipitation assay]). The factors' salt elution (lower or higher) from nonilluminated nuclei is consistent with the inverse of the FRAP results (faster or slower) and indicate that the FRAP kinetics for FoxA1 studied here primarily reflect intrinsic chromatin-binding stability. By these different experimental approaches, we conclude that the high intrinsic chromatin-binding capacity of the FoxA DBD is counteracted by a segment of the CTD to differentiate FoxA from linker histone with regard to nuclear mobility.

\section{RETENTION OF FOX TRANSCRIPTION FACTORS AND LINKER HISTONE IN MITOTIC CHROMATIN}

Long ago it was appreciated that the genome becomes transcriptionally silent during mitotic chromosome condensation and that RNA polymerase itself is not bound to mitotic chromatin (Prescott and Bender 1962; Gottesfeld and Forbes 1997; Spencer et al. 2000). Furthermore, many, if not most, transcription factors and chromatinbinding proteins are released from condensed chromosomes during mitosis (Egli et al. 2008). Yet a subset of transcription factors is known to be stably bound to the chromatin during mitosis, in a process referred to as "mitotic bookmarking" (Martinez-Balbas et al. 1995; Michelotti et al. 1997; Young et al. 2007). Mitotic bookmarking of transcription factors can allow for more rapid reactivation of target genes as cells exit mitosis (Blobel et al. 
2009; Dey et al. 2009). Previous studies of FoxI1 (Yan et al. 2006) and FoxA1 (Zaret et al. 2008) showed that the factors could be detected in mitotic chromatin and that FoxM1 is directly required for proper chromosome segregation (Alvarez et al. 2001; Laoukili et al. 2005). Thus, the special nucleosome- and chromatin-binding properties of Fox factors may make them generally appropriate as mitotic bookmarking factors.

Although the above studies characterized Fox factors bound to metaphase chromosomes of nocadazole-arrested cells, it was necessary to determine whether the factors could remain on the chromosomes throughout mitosis. To this end, we first transfected HUH7 hepatoma cells with a plasmid encoding GFP-FoxA, along with plasmids encoding GFP-linker histone H1o and GFP-NLS (SV40 nuclear localization signal), as positive and negative controls, respectively. We used nocodazole to block the transfected cells in metaphase, then washed out the drug and monitored GFP fluorescence in the cells as they progressed into anaphase and telophase. The location of the chromosomes was visualized by DAPI staining. As seen in Figure 4, GFP-FoxA1 clearly remained on the mitotic chromatin at all stages assayed, i.e., throughout mitosis (see arrows). In comparison, GFP-NLS was largely excluded from the mitotic chromatin and GFP-H1o remained bound, as expected (Fig. 4, arrows). These studies demonstrate that most or all of the GFP-FoxA1 remains bound to the chromosomes during mitosis.

To address whether untagged, endogenous FoxA1 remains bound to mitotic chromatin and does so at specific target sites, we performed a nocodazole arrest on nontransfected HUH7 cells, collected mitotic and asynchro- nous cells, and performed chromatin immunoprecipitation (ChIP) for FoxA1. As a control, we performed ChIP with nonimmune $\operatorname{IgG}$. The immunoprecipitation products were subjected to quantitative PCR (qPCR) for known and control FoxA1 target sequences. As seen in Figure 5, the transthyretin gene promoter (Ttr), which contains a highaffinity functional target site for FoxA1 (Costa et al. 1988), immunoprecipitates FoxA1 significantly over the IgG control in both the asynchronous and mitotic samples. The albumin enhancer (alb), which also contains functional FoxA1-binding sites (Liu et al. 1991), exhibited diminished binding in the mitotic sample, compared to the asynchronous sample. FoxA1 exhibited no binding to the control genomic site without a target sequence (Chr13). From these studies, we conclude that FoxA can bind to at least a subset of its functional target sites in mitotic cells.

\section{FINAL REMARKS}

Given that the DBD structure of FoxA factors so closely resembles that of linker histone, in this and previous studies, we compared how the two factors bind to chromatin in living cell nuclei, both from the perspective of nuclear mobility in interphase cells and of chromosome binding in mitotic cells. In both cases, FoxA has characteristics that much more closely resemble that of linker histone than for other transcription factors. However, FoxA factors recognize specific target sites in chromatin, whereas linker histones lack the corresponding amino acid residues that provide such binding and are well known to bind nonspecifically throughout genomic chromatin (Zhou et al. 1998). Thus, the FoxA factors, and likely the Fox family

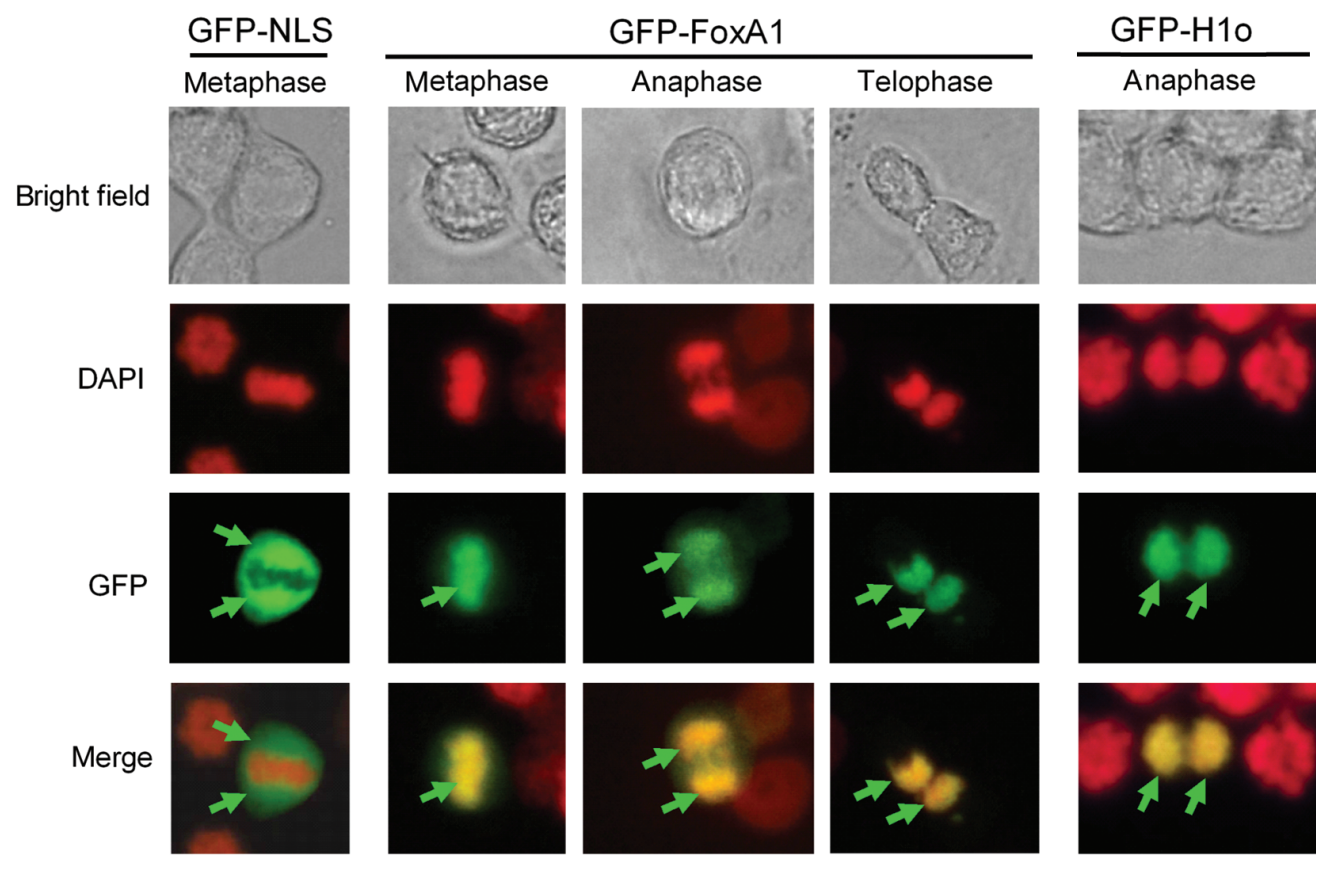

Figure 4. GFP-FoxA1 remains bound to chromosomes throughout mitosis. HUH7 hepatoma cells were transfected with plasmids encoding GFP-NLS (SV40 nuclear localization signal) as a negative control and GFP-FoxA1 and GFP-H1o (linker histone) as a positive control. Two days later, cells were treated with $0.06 \mu \mathrm{g} / \mathrm{mL}$ nocodazole. After $18 \mathrm{~h}$, the majority of cells were blocked in metaphase. Cells were then washed free of the drug and returned to culture for 45 min (GFP-NLS and GFP-H1o) or 75 min (GFP-FoxA1) to allow mitotic progression. Cells were fixed, stained with DAPI to visualize the chromosomes, and then imaged for DAPI and GFP fluorescence. 

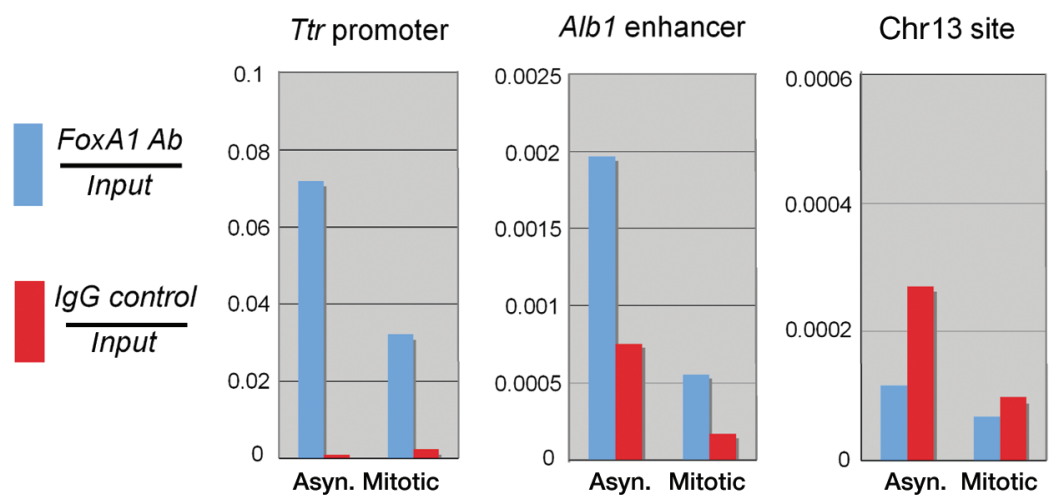

Figure 5. FoxA1 binds specific genomic sites in asynchronous and mitotic cells. HUH7 cells were treated for $24 \mathrm{~h}$ with 2 mM thymidine and then blocked in metaphase with $0.06 \mu \mathrm{g} / \mathrm{mL}$ nocodazole for $18 \mathrm{~h}$. Control and blocked cultures were treated with $1 \%$ formaldehyde for $20 \mathrm{~min}$ at room temperature. Cross-linked chromatin was then prepared, sonicated, and subjected to ChIP using FoxA1 antibodies (Abcam ab23738) or control IgG (Abcam ab46540). Products were subjected to qPCR reactions using primer sets specific for the human Ttr promoter (F: ATAGAACGAATGTTCCGATGCT, R: TCTCCTGAGCTAGGCTGCTTAT) and Alb1 enhancer (F: GCCCTGTCAACAAGAATGCTAT, R: CAAATGGCTGTGTTGATGAGTC), which contain functional FoxA1-binding sites as well as a negative control site on Chr13: 27288389-539 (F: TCTGTGTCCTTCACTGACATTAGAC, R: CTGCAACTGCTTACTAACTCATTCA). Data shown are averages from three asynchronous cell samples and two mitotic cell samples. Data shown are averages from three synchronous cell samples (Asyn.) and two mitotic cell samples (Mitotic).

in general, may represent an intermediate between the linker histones and typical transcription factors with regard to their chromatin scanning and binding properties.

What further distinguishes FoxA factors from linker histone? We found that FoxA's carboxy-terminal domain enhances the mobility of FoxA 1 in bulk chromatin, so that the factor does not move as slowly as linker histone. This mobilizing domain may aid FoxA function as a transcription factor, allowing it to search for target sites, whereas the linker histones function as a more stable chromatin component. Another function of the FoxA carboxy-terminal domain is to open compacted chromatin at specific binding sites, by interacting with core histones and possibly disrupting local internucleosomal interactions (Cirillo et al. 2002). Preliminary deletion studies of the FoxA carboxy-terminal domain indicate that the chromatin opening and chromatin mobility functions are separable (T Sekiya and $\mathrm{K}$ Zaret, unpubl.).

In conclusion, both the carboxy-terminal domain and the ability of the FoxA DBD to recognize specific target sequences help distinguish the factor from a linker histone. By these means, the transcription factor retains fundamental chromatin-binding properties but with different nuclear movement and chromatin access associated with the early chromatin-binding functions of a pioneer factor.

\section{ACKNOWLEDGMENTS}

Zaret laboratory research described in this chapter was supported by grants from the National Institutes of Health (R01-DK82623, R37-GM36477).

\section{REFERENCES}

Alvarez B, Martinez AC, Burgering BM, Carrera AC. 2001. Forkhead transcription factors contribute to execution of the mitotic programme in mammals. Nature 413: 744-747.

Blobel GA, Kadauke S, Wang E, Lau AW, Zuber J, Chou MM,
Vakoc CR. 2009. A reconfigured pattern of MLL occupancy within mitotic chromatin promotes rapid transcriptional reactivation following mitotic exit. Mol Cell 36: 970-983.

Cirillo LA, Zaret KS. 1999. An early developmental transcription factor complex that is more stable on nucleosome core particles than on free DNA. Mol Cell 4: 961-969.

Cirillo LA, McPherson CE, Bossard P, Stevens K, Cherian S, Shim E-Y, Clark EA, Burley SK, Zaret KS. 1998. Binding of the winged-helix transcription factor HNF3 to a linker histone site on the nucleosome. EMBO J 17: 244-254.

Cirillo L, Lin FR, Cuesta I, Jarnik M, Friedman D, Zaret K. 2002. Opening of compacted chromatin by early developmental transcription factors HNF3 (FOXA) and GATA-4. Mol Cell 9: 279-289.

Clark KL, Halay ED, Lai E, Burley SK. 1993. Co-crystal structure of the HNF3/fork head DNA recognition motif resembles histone H5. Nature 364: 412-420.

Costa RH, Grayson DR, Xanthopoulos KG, Darnell JE Jr. 1988. A liver-specific DNA-binding protein recognizes multiple nucleotide sites in regulatory regions of transthyretin, $\mathrm{a}_{\text {-anti- }}$ trypsin, albumin, simian virus 40 genes. Proc Natl Acad Sci 85: 3840-3844.

Cuesta I, Zaret KS, Santisteban P. 2007. The forkhead factor FoxE1 binds to the thyroperoxidase promoter during thyroid cell differentiation and modifies compacted chromatin structure. Mol Cell Biol 27: 7302-7314.

Dey A, Nishiyama A, Karpova T, McNally J, Ozato K. 2009. Brd4 marks select genes on mitotic chromatin and directs postmitotic transcription. Mol Biol Cell 20: 4899-4909.

Egli D, Birkhoff G, Eggan K. 2008. Mediators of reprogramming: Transcription factors and transitions through mitosis. Nat Rev Mol Cell Biol 9: 505-516.

Gottesfeld JM, Forbes DJ. 1997. Mitotic repression of the transcriptional machinery. Trends Biochem Sci 22: 197-202.

Gualdi R, Bossard P, Zheng M, Hamada Y, Coleman JR, Zaret KS. 1996. Hepatic specification of the gut endoderm in vitro: Cell signaling and transcriptional control. Genes Dev 10: 1670-1682.

Hager GL, McNally JG, Misteli T. 2009. Transcription dynamics. Mol Cell 35: 741-753.

Hannenhalli S, Kaestner KH. 2009. The evolution of Fox genes and their role in development and disease. Nat Rev Genet 10: 233-240.

Hatta M, Cirillo LA. 2007. Chromatin opening and stable perturbation of core histone:DNA contacts by FoxO1. J Biol Chem 282: 35583-35593. 
Hinow P, Rogers CE, Barbieri CE, Pietenpol JA, Kenworthy AK, DiBenedetto E. 2006. The DNA binding activity of p53 displays reaction-diffusion kinetics. Biophys J 91: 330-342.

Karpova TS, Chen TY, Sprague BL, McNally JG. 2004. Dynamic interactions of a transcription factor with DNA are accelerated by a chromatin remodeller. EMBO Rep 5: 1064-1070.

Kimura H, Cook PR. 2001. Kinetics of core histones in living human cells: Little exchange of $\mathrm{H} 3$ and $\mathrm{H} 4$ and some rapid exchange of H2B. J Cell Biol 153: 1341-1353.

Laoukili J, Kooistra MR, Bras A, Kauw J, Kerkhoven RM, Morrison A, Clevers H, Medema RH. 2005. FoxM1 is required for execution of the mitotic programme and chromosome stability. Nat Cell Biol 7: 126-136.

Lee CS, Sund NJ, Behr R, Herrera PL, Kaestner KH. 2005. Foxa2 is required for the differentiation of pancreatic $\alpha$-cells. Dev Biol 278: 484-495.

Liu JK, DiPersio CM, Zaret KS. 1991. Extracellular signals that regulate liver transcription factors during hepatic differentiation in vitro. Mol Cell Biol 11: 773-784.

Luger K, Mäder AW, Richmond RK, Sargent DF, Richmond TJ. 1997. Crystal structure of the nucleosome core particle at 2.8 Å resolution. Nature 389: 251-255.

Martinez-Balbas MA, Dey A, Rabindran SK, Ozato K, Wu C. 1995. Displacement of sequence-specific transcription factors from mitotic chromatin. Cell 83: 29-38.

McNally JG, Muller WG, Walker D, Wolford R, Hager GL. 2000. The glucocorticoid receptor: Rapid exchange with regulatory sites in living cells. Science 287: 1262-1265.

Michelotti EF, Sanford S, Levens D. 1997. Marking of active genes on mitotic chromosomes. Nature 388: 895-899.

Misteli T, Gunjan A, Hock R, Bustin M, Brown DT. 2000. Dynamic binding of histone $\mathrm{H} 1$ to chromatin in living cells. Nature 408: 877-881.

Mueller F, Wach P, McNally JG. 2008. Evidence for a common mode of transcription factor interaction with chromatin as revealed by improved quantitative fluorescence recovery after photobleaching. Biophys J 94: 3323-3339.

Nunez E, Kwon YS, Hutt KR, Hu Q, Cardamone MD, Ohgi KA, Garcia-Bassets I, Rose DW, Glass CK, Rosenfeld MG, et al. 2008. Nuclear receptor-enhanced transcription requires motorand LSD1-dependent gene networking in interchromatin granules. Cell 134: 189.

Pani L, Overdier DG, Porcella A, Qian X, Lai E, Costa RH. 1992. Hepatocyte nuclear factor $3 \mathrm{~b}$ contains two transcriptional activation domains, one of which is novel and conserved with the Drosophila fork head protein. Mol Cell Biol 12: 37233732 .
Phair RD, Scaffidi P, Elbi C, Vecerova J, Dey A, Ozato K, Brown DT, Hager G, Bustin M, Misteli T. 2004. Global nature of dynamic protein-chromatin interactions in vivo: Three-dimensional genome scanning and dynamic interaction networks of chromatin proteins. Mol Cell Biol 24: 6393-6402.

Prescott DM, Bender MA. 1962. Synthesis of RNA and protein during mitosis in mammalian tissue culture cells. Exp Cell Res 26: 260-268.

Ramakrishnan V, Finch JT, Graziano V, Lee PL, Sweet RM. 1993. Crystal structure of globular domain of histone $\mathrm{H} 5$ and its implications for nucleosome binding. Nature 362: 219-224.

Schalch T, Duda S, Sargent DF, Richmond TJ. 2005. X-ray structure of a tetranucleosome and its implications for the chromatin fibre. Nature 436: 138-141.

Sekiya T, Muthurajan UM, Luger K, Tulin AV, Zaret KS. 2009. Nucleosome-binding affinity as a primary determinant of the nuclear mobility of the pioneer transcription factor FoxA. Genes Dev 23: 804-809.

Simpson RT, Thoma F, Brubaker JM. 1985. Chromatin reconstituted from tandemly repeated cloned DNA fragments and core histones: A model system for study of higher order structure. Cell 42: 799-808.

Spencer CA, Kruhlak MJ, Jenkins HL, Sun X, Bazett-Jones DP. 2000. Mitotic transcription repression in vivo in the absence of nucleosomal chromatin condensation. J Cell Biol 150: 13-26.

Vallet V, Antoine B, Chafey P, Vandewalle A, Kahn A. 1995. Overproduction of a truncated hepatocyte nuclear factor 3 protein inhibits expression of liver-specific genes in hepatoma cells. Mol Cell Biol 15: 5453-5460.

Yan J, Xu L, Crawford G, Wang Z, Burgess SM. 2006. The forkhead transcription factor FoxI1 remains bound to condensed mitotic chromosomes and stably remodels chromatin structure. Mol Cell Biol 26: 155-168.

Young DW, Hassan MQ, Pratap J, Galindo M, Zaidi SK, Lee SH, Yang X, Xie R, Javed A, Underwood JM, et al. 2007. Mitotic occupancy and lineage-specific transcriptional control of rRNA genes by Runx2. Nature 445: 442-446.

Zaret KS, Dipersio CM, Jackson DA, Montigny WJ, Weinstat DL. 1988. Conditional enhancement of liver-specific gene transcription. Proc Natl Acad Sci 85: 9076-9080.

Zaret KS, Watts J, Xu J, Wandzioch E, Smale ST, Sekiya T. 2008. Pioneer factors, genetic competence, and inductive signaling: Programming liver and pancreas progenitors from the endoderm. Cold Spring Harb Symp Quant Biol 73: 119-126.

Zhou Y-B, Gerchman SE, Ramakrishnan V, Travers A, Muyldermans S. 1998. Position and orientation of the globular domain of linker histone H5 on the nucleosome. Nature 395: 402-405. 


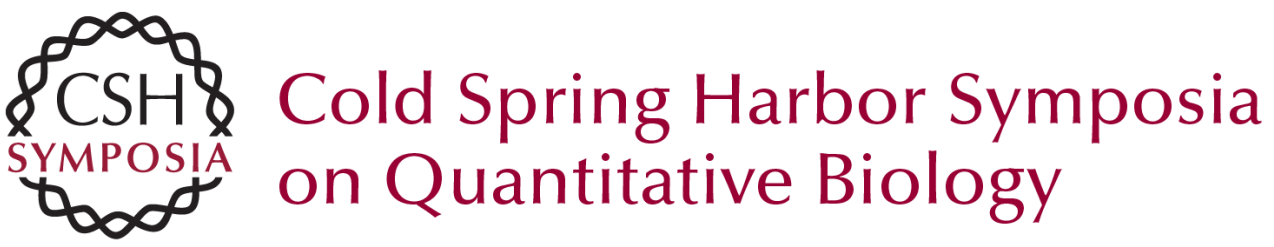

\section{Nuclear Mobility and Mitotic Chromosome Binding: Similarities between Pioneer Transcription Factor FoxA and Linker Histone $\mathrm{H} 1$}

K.S. Zaret, J.M. Caravaca, A. Tulin, et al.

Cold Spring Harb Symp Quant Biol 2010 75: 219-226 originally published online April 18, 2011 Access the most recent version at doi:10.1101/sqb.2010.75.061

References This article cites 42 articles, 17 of which can be accessed free at: http://symposium.cshlp.org/content/75/219.full.html\#ref-list-1

\section{License}

Email Alerting Receive free email alerts when new articles cite this article - sign up in Service the box at the top right corner of the article or click here. 